\title{
B-cell targeted therapy in systemic lupus erythematosus: potential of rituximab
}

\author{
This article was published in the following Dove Press journal: \\ Biologics:Targets and Therapy \\ 25 September 2012 \\ Number of times this article has been viewed
}

\section{E Wiesik-Szewczyk M Olesinska}

Institute of Rheumatology, Department of Connective Tissue Diseases, Warsaw, Poland

Correspondence: Ewa Wiesik-Szewczyk Institute of Rheumatology, Department of Connective Tissue Disease,

02-637 Warsaw, Poland

Tel +48228445726

Fax +48 226467894

Email ewa.w.szewczyk@gmail.com

\begin{abstract}
Systemic lupus erythematosus (SLE) is a complex autoimmune disease of unknown etiology, and the limited available therapeutic options for this disease, are frustrating to both clinicians and patients. However, recent advances in the understanding of disease mechanisms have given rise to numerous studies on specific approaches to SLE treatment. Rituximab, the first chimeric, mouse-human monoclonal antibody which is directed against CD20, seems to be a new therapeutic option. The purpose of this review is to explain the current clinical evidence on the therapeutic use of rituximab in adult SLE patients. Two randomized clinical trials with rituximab (the EXPLORER and LUNAR studies) failed to prove efficacy of this drug on SLE. Ongoing data analysis continues to explain the reasons behind why this treatment fails to work. However data from open source and observational studies contrast with clinical trials results. The global analysis of this data supports the off-label use of rituximab in subsets of SLE that are refractory to standard treatment.
\end{abstract}

Keywords: B cells, systemic lupus erythematosus, rituximab, off-label use, clinical trials

\section{SLE, overview of the diseases}

Systemic lupus erythematosus (SLE) is a chronic autoimmune disease with a heterogeneous clinical picture and an unpredictable prognosis. Clinical symptoms include arthralgia, arthritis, skin rashes, serositis, hematological abnormalities, and can lead to severe central nervous system dysfunction and renal inflammation, which can ultimately result in renal insufficiency that requires dialysis. The clinical symptoms are accompanied with immunologic abnormalities that include the presence of antinuclear antibodies, antibodies that are specific to double-stranded deoxyribonucleic acid, and the Smith antigen. Complement activation including the presence of circulating complement split products and depressed levels of C3 and C4 complement in the serum accompany serological abnormalities. ${ }^{1}$

Due to protean clinical presentation and the lack of a unique confirmatory test, diagnosis of SLE remains a challenge. To identify patients in clinical studies, the American College of Rheumatology criteria for the classification of SLE are used (Table 1). ${ }^{2,3}$ A person has SLE if at least 4 of the 11 criteria are present either simultaneously or serially during an observation period. Criteria 1-9 are clinical, while 10 and 11 pertain to a positive serological test. Patients included in Phase III clinical trials have had to fulfill at least one of the immunological criteria.

Standard of care therapy for SLE typically involves antimalarials, combined with corticosteroids and non-steroidal anti-inflammatory drugs. In more 
Table I The revised criteria for the classification of systemic lupus erythematosus

Criteria for the classification of systemic lupus erythematosus
Clinical criteria
I. Malar rash
2. Discoid rash
3. Photosensitivity
4. Oral ulcers
5. Arthritis
6. Serositis
7. Renal disorder
8. Neurological disorder
9. Hematological disorder
Immunological criteria
I0. Anti-DNA
Anti-Sm
$\quad$ Anti-phospholipids
Lupus anticoagulant
False positive serological test for syphilis
II. Abnormal titer of ANA

Abbreviations: DNA, deoxyribonucleic acid; Sm, Smith antigen; ANA, antinuclear antibodies.

severe cases, when there is more internal organ involvement or corticosteroid-dependence, non-selective immunomodulatory and immunosuppressive agents are used, such as azathioprine, methotrexate, mycophenolate mofetil (MMF), cyclophosphamide, and cyclosporine. For severe, refractory, or recurrent diseases, combinations of therapies might be mandatory.

Current therapies are often ineffective in sustaining remission of the disease. They may also have to be administered over a prolonged time period and in doses associated with substantial adverse events and drug-related complications. In long-term follow-up, the majority of patients suffer from organ damage. Still, mortality rates among SLE patients are higher than among healthy populations. The increased mortality rate is largely a function of the lupus itself, resultant infections, and in later stages can be associated with cardiovascular complications. This bimodal pattern of lupus mortality rates was recognized 30 years ago and remains unchanged. ${ }^{4}$ Therefore, treatments that involve lower doses of corticosteroids, or therapies that are more effective and better tolerated than cytotoxic drugs are needed.

In the past decade, biologics targeting immune cells, costimulatory pathways, and selected cytokines, or their receptors have been approved for the treatment of a wide variety of autoimmune diseases. Surprisingly, the results of clinical trials have been disappointing for SLE, a set of prototypic B-cell dependent diseases. ${ }^{5}$ The exception to this has been belimumab, a B-cell activating factor inhibitor, which was the first new drug in 50 years to be approved for SLE treatment; however, the clinical efficacy of belimumab seems to be modest. ${ }^{6}$

The other B-cell targeted agent, rituximab, failed to meet primary end-points in two clinical trials, but is widely used in an off-label manner. This review will focus on the potential of rituximab in the treatment of SLE patients in clinical practice.

\section{SLE and B lymphocytes}

B cells play a crucial pathologic role in SLE. ${ }^{7}$ Abnormal B cell proliferation, maturation, prolonged life-span of auto reactive clones, and autoantibody production are documented along with immune deregulation and tolerance breakdown. ${ }^{8,9}$ Some SLE patients have abnormal selection at the immature to early transitional stage due to intrinsic B cell defects. ${ }^{10}$ Auto reactive B cells in SLE differentiate into memory and plasma cells. In immature bone marrow, diminished auto reactive $\mathrm{B}$ cell deletion is associated with polymorphisms in gene encoding modulators of BCR signaling, such as PTPN22 and BLK. ${ }^{11,12}$ In antigen activated mature B cells, differentiation to effector cells is stimulated by $\mathrm{T}$ cell help and costimulation, enhanced BCR signals via CD19 upregulation, increased TLR signals, or impaired negative signals mediated trough FcyRIIB and CD22. ${ }^{13}$ Abnormal B cell function is characterized by the production of autoantibodies - a serologic hallmark of SLE. Another abnormal B cell function is cytokine secretion. B cells have been shown to produce interleukin- $4,6,10$, interferon gamma, transforming growth factor beta, and limphotoxin alfa. The overproduction of cytokines in SLE can lead to amplification of autoimmune response. ${ }^{8} \mathrm{~B}$ cells are also antigen presenting cells; B cells activate $\mathrm{T}$ cells by surface expression of peptide-MHC complex that interact with $\mathrm{T}$ cell receptors. ${ }^{14}$

In summary B cells play a crucial role in SLE pathogenesis through the presentation of self-antigens, T cell activation, and through the production of autoantibodies and cytokines. All of the above have provided the rationale for the use of B cell directed therapy in SLE treatment.

Rituximab is the first chimeric, mouse-human monoclonal antibody which is directed against CD20. CD20 is a B lymphocyte restricted surface molecule that is expressed from pre-B to memory B cells. Despite intensive studies, the precise function of rituximab remains puzzling. CD20-knockout mice do not present with specific phenotype abnormalities and seem to preserve 
normal immunologic response, and in addition, CD20 has no known natural ligand. ${ }^{15}$ It is a phosphoprotein with a structure of four transmembrane regions and an amino-acid extracellular loop. According to structural homologies, it is supposed that CD20 may have a calcium channel function. Administration of the CD20-specific antibody results in the death of B cells, which is achieved by antibody-dependent cell-mediated cytotoxicity, complement-mediated lyses, or apoptosis.

Rituximab does not target pre-B cells, plasmablasts, or plasma cells producing disease-specific autoantibodies (double-stranded deoxyribonucleic acid or anti-Smith antigens), which can lead to diminished efficacy of the drug in patients with B cell depletion who demonstrate poor serologic response. On the other hand, the decline of autoantibodies is not always associated with a good clinical response. It seems reasonable that different symptoms of SLE result from different mechanisms and are not always autoantibody-dependent.

Rituximab influences homeostasis and improves the disturbances found in peripheral B cells that are characteristic of active SLE, and it affects both the cellular and the humoral arm of the immune system. ${ }^{16,17}$ After effective $B$ cell depletion during a reconstitution period, naive $\mathrm{B}$ cell lymphopenia, expansion of a CD27-, IgD- (double negative) population, and expansion of circulating plasmablasts are significantly decreased. In addition, the frequency of auto reactive memory B cells was found to be decreased 1 year post-treatment. ${ }^{16}$ However, the magnitude, duration, and consequences of depletion therapy in SLE have not yet been completely elucidated. Long-term follow-up (mean duration 41 months) has shown a delayed recovery of memory CD27+ B cells in peripheral blood and lymphoid tissue after rituximab administration. ${ }^{18}$

B cell levels after rituximab administration have been measured in clinical trials, but their importance in clinical practice has not been proven. The grade of B cell depletion by routine measures was not predictive for a clinical response. It was suggested that the timing of retreatment for patients with rheumatoid arthritis and vasculitis should be based mainly on clinical activity. However, measurement by highly sensitive flow cytometry, which can define B cell numbers 50-100 times lower than conventional techniques, predicts the overall effectiveness of rituximab. In a recent study of 39 patients with active SLE treated with rituximab, clinical outcomes correlated with the level of B cell depletion. Moreover, plasmablast repopulation was significantly faster in patients with earlier relapse when compared to patients with later relapse. ${ }^{19}$ In clinical practice, the regimen and tools for B cell depletion assessment in SLE patients have yet to be determined.

\section{Clinical trials EXPLORER STUDY - Rituximab in moderately to severely active extra-renal SLE}

The aim of a placebo-controlled, double-blind, multicenter study was to assess the efficacy and safety of rituximab in patients with moderately or severely active extra-renal SLE. ${ }^{20}$ All subjects included into the study had to fulfill the American College of Rheumatology classification criteria, including a positive test for antinuclear antibodies. Activity of the disease was defined using the British Isles Lupus Assessment Group (BILAG) organ system, which scores patients based on the need for alterations and intensification of therapy. Severe disease was indicated if more than one organ system was scored as A, and a moderate form of the disease was indicated if at least two organ systems received a B score. Patients received a stable dose of one immunosuppressive drug at entry, which was maintained during the trial.

Exclusion criteria involved patients with severe central nervous system or organ-threatening lupus, or any conditions requiring significant use of steroids, or recent treatment with cyclophosphamide or calcineurin inhibitors.

Patients were randomized at a 2:1 ratio to receive either rituximab or placebo on days $1,15,168$, and 182 , and these were added to their current standard of care therapy: the baseline immunosuppressive regimen (azathioprine, mycophenolat mofetil, or methotrexate) and prednisone, given according to the protocol (daily dose at least $0.5 \mathrm{mg} / \mathrm{kg}$ ) to achieve immediate control of symptoms.

Primary endpoints were clinical response defined as achieving BILAG C scores or better in all organs at week 24 without experiencing a severe flare from day 1 to week 24 , and maintaining this response to week 52 . Treatment failure was noted upon one new BILAG B score after 6 months, which is very rigorous.

The trial enrolled 257 patients with significant disease activity $(81 \%$ entered with $1 \geq$ BILAG A score or $3 \geq$ BILAG B score), mostly in mucocutaneous or musculoskeletal systems, and with constitutional features. It should be noted that safety, tolerability, and the patient dropout rate was similar across both patients receiving placebo and those receiving rituximab. 
No differences were observed between placebo and rituximab in the efficacy end-points. In both groups, significant improvement was observed by day 28 due to initial steroid treatment and was maintained after dose tapering. However in the African American/Hispanic group, which comprised one-third of patients, the clinical response differed significantly between the placebo and treatment arms $(P=0.0408)$, which suggested the beneficial effect of rituximab in this subgroup.

Further evaluation of patient subsets and biomarkers has since continued. Recently, exploratory reanalysis of data from the EXPLORER study was conducted, considering alternative definitions for flare. ${ }^{21}$ The paper analyzed patients who achieved low disease activity (BILAG C or D) at any point prior to week 52 . The following variables were assessed: time to first severe flare ( $\geq 1 \mathrm{~A}$ BILAG score or $\geq 3$ B BILAG scores), time to first A BILAG flare, and the number of $A$ flares per patient per year. No difference was observed between those taking rituximab and placebo in preventing or delaying flares when accounting for both severe and moderate flares. However, those in the rituximab group demonstrated a longer time to the first A flare as well as a significant decrease of A flares per patient per year compared with those in the placebo group. In summary, the authors stated that no conclusion about rituximab efficacy can be drawn. The data suggest that rituximab may lessen severe flares defined by BILAG A score. Moreover, the data confirm the necessity for the revision in design of future clinical studies. The analyses based on BILAG A flares may be more specific and clinically significant.

Another possibility is use of compound variables to assess patient outcomes, as performed in the Phase III studies of belimumab. ${ }^{22,23}$ The new robust Systemic Lupus Erythematosus Responder Index assesses improvements in disease activity without worsening the overall condition or the development of significant diseases activity in new organ systems. The Responder Index response is defined as (1) a $\geq 4$-point reduction in the SELENA-SLEDAI score; (2) no new BILAG A, or no more than one new BILAG B domain score; and (3) no deterioration from baseline in the physician's global assessment by $\geq 0.3$ points. ${ }^{24}$

The EXPLORER trial accomplished some important tasks including enrolling demonstrably ill patients, setting strict background rules for therapy, providing clear definitions of efficacy endpoints, and identifying treatment failure cut-off sensitivity points. Negative results suggest that the disease is more biologically heterogeneous and is not uniquely B-cell driven. Moreover the methods used to rate clinical activity were probably not optimal. The trial also did not examine the possibility of synergic use of cyclophosphamide, which was one of exclusion criteria.

\section{LUNAR STUDY - rituximab in active proliferative lupus nephritis}

The aim of this study was to assess whether the addition of rituximab to a background of MMF plus corticosteroids is beneficial in patients with proliferative lupus nephritis. ${ }^{25}$ Patients were eligible if they were diagnosed with SLE according to the American College of Rheumatology criteria and had a history of positive antinuclear antibodies. Patients required a diagnosis of lupus nephritis (LN) that was supported by both renal biopsy and proteinuria (presenting with a urine/protein/creatinine ratio $>1$ ). If the biopsy was performed $>3$ months before screening, the collection of active urinary sediment was also required.

Patients were randomized 1:1 to receive either placebo or rituximab. MMF was initiated at $1.5 \mathrm{~g}$ /day and increased to $3 \mathrm{~g} /$ day by week 4 , and maintained through the study. Methylprednisolone $1000 \mathrm{mg}$ was administered intravenously prior to administration of the study drug on day 1 , and across the consecutive 3 days as therapy for active LN. Subsequently, oral prednisone was given $(0.75 \mathrm{mg} / \mathrm{kg} /$ day $)$ until day 16 , and tapered to $\leq 10 \mathrm{mg}$ /day by week 16 . Other immunosuppressive agents were not allowed. Any new immunosuppressants or the introduction of high dose corticoids required that participants discontinued their participation in the study, and subjects were identified as experiencing treatment failure.

The primary efficacy endpoint was renal response, defined as complete renal response (CRR), partial renal response (PRR), or no response at week 52 (Table 2). Secondary endpoints were CRRs sustained from week 24 through 52, CRR rates at week 52, reduction in baseline urine/protein/creatinine (UPC) from $>3.0$ to $<1.0$ at week 52 and time to first CRR.

The trial enrolled 144 patients. Sixty nine percent of the patients were first diagnosed with $\mathrm{LN}$ within 2 years of randomization, and half experienced their first episode of LN. Renal response rate (CRR, partial renal response, or no response) at week 52 was not statistically different between the rituximab and the placebo group. $(P=0.55)$. Again, as in the EXPLORER trial, a prespecified subgroup analysis of the overall renal response revealed that at week 52, black patients treated with rituximab had a higher response rate than the placebo group ( $70 \%$ vs $45 \%$ ). Regarding secondary endpoints, rituximab exhibited a reduction of UPC at week 52 , and the difference between the two groups was statistically significant at week $78(P<0.04)$. Similarly, the rituximab 
Table 2 Criteria for renal response in the LUNAR study

\begin{tabular}{ll}
\hline Renal response & Criteria \\
\hline Complete renal response & Normal serum creatinine if abnormal \\
(CRR) & at study entry; or \\
& Serum creatinine $\leq$ I I5\% if normal \\
& at study entry; and \\
& Inactive urinary sediment ( $<5 \mathrm{RBC} / \mathrm{HPF}$ \\
& and absence of RBC casts); and \\
& Urine/creatinine/protein ratio $<0.5$ \\
& Serum creatinine $\leq$ II $5 \%$ if normal at \\
& study entry; \\
Partial renal response & RBC/HPF $\leq 50 \%$ above baseline and \\
(PRR) & absence of RBC cast; \\
& At least $50 \%$ decrease in UPC to $<1.0$ \\
& if baseline UPC was $\leq 3.0 ;$ or \\
& To $\leq 3.0$ if baseline UPC was above 3.0 \\
& Criteria for CRR or PRR not met; or \\
& Early termination; or \\
Inability to assesses endpoint due to \\
mo response & missing data; or \\
& Initiation of new immunosuppressant; or \\
& Corticosteroid rescue therapy \\
\hline
\end{tabular}

Abbreviations: LUNAR, Lupus Nephritis Assessment with Rituximab; RBC/HPF, red blood cells per high power field; RBC, red blood cells; UPC, urine/protein/ creatinine ratio; CRR, complete renal response; PRR, partial renal response.

group was more likely to achieve CRR with respect to proteinuria at week $78(P=0.04)$. There were no statistically significant differences in other secondary endpoints.

LUNAR is the largest randomized placebo controlled study to evaluate the effect of rituximab added to initial therapy for active proliferative LN. The study outcomes did not meet primary efficacy endpoints, although there were more PRR in the rituximab group. These data are in disagreement with previous uncontrolled studies of $\mathrm{LN}$ that had shown favorable responses to rituximab. However, it is important to note that these open studies enrolled patients who were mainly refractory to therapies with cyclophosphamide or MMF; LUNAR excluded such patients. Moreover, half of these patients had their first episode of LN in the LUNAR trial.

\section{Off-label clinical experience}

Uncontrolled clinical studies with rituximab have shown promising results regardless of medication regimens, tools used for assessment, and indications (overall SLE activity, ${ }^{26-38}$ SLE with hemolytic anemia, ${ }^{39}$ refractory SLE, ${ }^{40-42}$ lupus nephritis, ${ }^{43-48}$ or severe central nervous system involvement). ${ }^{49,50}$ Results of published clinical studies that enrolled at least 10 patients are listed in Table 3. A systematic review of off-label rituximab use in refractory SLE documented a significant improvement in at least one organ manifestation in majority of patients $(90 \%) .{ }^{47}$ The French Autoimmunity and Rituximab Registry presented data patients treated with rituximab in regular clinical practice. ${ }^{37}$ Overall response was defined by SELENA-SLEDAI reduction $\geq 3$. An organ-system response was defined as a $50 \%$ improvement (partial response) or disappearance of baseline manifestations (complete response). The study involved 136 patients with mild to severe SLE (the mean SELENASLEDAI $=11.3$ ). A total of 42 patients had LN. Rituximab was added to a stable background immunosuppressive therapy (59 patients) or given in combination with new immunosuppressive agents (12 patients). The mean duration of follow-up was 18 months.

SELENA-SLEDAI reduction was observed in 80 of 113 patients available for analysis (71\%). The most significant improvement was observed in articular, cutaneous, renal, and hematological symptoms, and patients achieved at least a $70 \%$ improvement in their symptoms (combined partial and complete response). Moreover, a statistically significant steroid-sparing effect was observed. Among the responders, $41 \%$ experienced a flare and of those, $90 \%$ subsequently responded to rituximab re-treatment. The authors concluded that analysis of data from the French registry showed that the efficacy of rituximab in SLE patients was significant in actual clinical practice. ${ }^{37}$

Recently pooled data from European cohorts diagnosed with biopsy-proven LN were published. ${ }^{41}$ Analysis involved 164 patients. In $82(50 \%)$ cases, rituximab was administered to patients with LN that was refractory to standard therapies; in $69(42 \%)$ cases, rituximab was provided for LN flare; and in 13 (8\%) cases, the drug was provided as first line therapy. Rituximab was administered in combination with steroids, cyclophosphamide ( $\mathrm{n}=58)$, or MMF $(\mathrm{n}=55)$. Significant improvements in proteinuria and a reduction in the protein/creatinine ratio were observed at 12 months $(P<0.001)$. Nephrotic syndrome and renal insufficiency at baseline predicted a worse response. The authors concluded that rituximab can be an effective option for patients with $\mathrm{LN}$, especially for those refractory to standard treatment or for those with recurrent flares. ${ }^{41}$

These encouraging results contrast with the poor outcomes reported from both clinical trials. These contradictory findings can be attributed to several reasons including patient selection. The open studies enrolled different populations treated with cyclophosphamide (those with refractory diseases or those with severe CNS). The next issue was the method used to assess clinical activity. The BILAG score is a transitional index developed for intention-to-treat analysis, and might not be perfect for use in regular clinical practice. ${ }^{51}$ There are many published analyses suggesting that 
Table 3 Open-label studies of rituximab in adult patients $(n \geq 10)$ with systemic lupus erythematosus

\begin{tabular}{|c|c|c|c|}
\hline Author & $\mathbf{N}$ of patients & Result & Comments \\
\hline \multicolumn{4}{|l|}{ Moderate to severe SLE } \\
\hline \multirow[t]{2}{*}{ Galarza-Maldonado et al ${ }^{26}$} & 46 & Effective & Latin-American Patients, \\
\hline & & & $\begin{array}{l}\text { Rituximab followed by hydroxychloroquine and MMF, } \\
50 \% \text { remission in } 24 \text { month }\end{array}$ \\
\hline \multirow[t]{3}{*}{ Catapano et $\mathrm{a}^{27}$} & 31 & Effective & $87 \%$ remission (partial or complete); \\
\hline & & & 30 months follow-up, \\
\hline & & & Relapses responded for re-treatment \\
\hline \multirow[t]{2}{*}{ Leandro et $\mathrm{al}^{28}$} & 24 & Effective & Patients refractory to conventional immunosuppressants \\
\hline & & & Global BILAG score improvement \\
\hline \multirow[t]{2}{*}{ Looney et $\mathrm{al}^{29}$} & 17 & Effective & SLAM score improvement, \\
\hline & & & 12 months follow-up \\
\hline \multirow[t]{2}{*}{$\mathrm{Ng}$ et $\mathrm{a}^{30}$} & 32 & Effective & Rituximab with cyclophosphomide \\
\hline & & & 36 months follow-up \\
\hline Cambridge et $\mathrm{al}^{31}$ & 25 & Effective & $\begin{array}{l}\text { Negative response correlation with expanded ANA profile } \\
\text { and BLyS concentration }\end{array}$ \\
\hline \multirow[t]{2}{*}{ Albert et $\mathrm{a}^{32}$} & 18 & Effective & Patients who failed at least one immunosuppressant \\
\hline & & & SLEDAI reduction \\
\hline \multirow[t]{2}{*}{ Reynolds et $\mathrm{al}^{33}$} & 11 & Effective & BILAG reduction, steroid sparing effect, positive effect \\
\hline & & & for interstitial lung disease \\
\hline Tanaka et al ${ }^{34}$ & 15 & Effective & BILAG score reduction \\
\hline Lu et $\mathrm{al}^{35}$ & 45 & Effective & 19 achieved complete remission, 21 achieved partial remission \\
\hline Garcia-Carrasco et al ${ }^{36}$ & 52 & Effective & Mexican population \\
\hline Terrier et $\mathrm{a}^{37}$ & 136 & Effective & Data from French Registry, patient from routine clinical practice \\
\hline Jónsdóttir et al ${ }^{38}$ & 16 & Effective & Rituximab with cyclophosphamide \\
\hline Gomard-Mennesson et $\mathrm{al}^{39}$ & 26 & Effective & Severe immune hemolytic anemia \\
\hline Lindholm et $\mathrm{a}^{40}$ & 31 & Effective & $\begin{array}{l}\text { Patients refractory to conventional immunosuppressive } \\
\text { treatment }\end{array}$ \\
\hline \multicolumn{4}{|l|}{ Lupus nephritis } \\
\hline Díaz-Lagares et $\mathrm{al}^{41}$ & 164 & Effective & $\begin{array}{l}\text { Biopsy proven } \mathrm{LN} \text {, pooled data from European cohort, } \\
\text { rituximab in combination with cyclophosphamide or MMF }\end{array}$ \\
\hline Lateef et $\mathrm{al}^{42}$ & 10 & Effective & Potential cost saving in LN \\
\hline Boletis et $\mathrm{al}^{43}$ & 10 & Effective & Rituximab with MMF in proliferative nephritis \\
\hline Melander et $\mathrm{al}^{45}$ & 20 & Effective & No response in rapidly progressive gromelunophritis \\
\hline \multirow[t]{2}{*}{ Jónsdóttir et al ${ }^{46}$} & 28 & Effective & Proliferative, membranous LN, data pooled from 2 centers, \\
\hline & & & Rituximab with cyclophosphamide \\
\hline Ramos-Casals et $\mathrm{a}^{47}$ & 164 & Effective & Systematic analysis of seven observational studies \\
\hline Pepper et $\mathrm{a}^{48}$ & 18 & Effective & Significant steroid sparing effect \\
\hline \multicolumn{4}{|c|}{ Central nervous system SLE } \\
\hline Tokunaga et $\mathrm{al}^{49}$ & 10 & Effective & Rapid response in NP SLE \\
\hline \multirow[t]{2}{*}{ Narváez et al ${ }^{50}$} & 35 & Effective & Clinical improvement; \\
\hline & & & Steroid sparing effect \\
\hline
\end{tabular}

Abbreviations: SLE, systemic lupus erythematosus; MMF, mycophenolate mofetil; BILAG, British Isles Lupus Assessment Group; SLAM, Systemic Lupus Activity Measure; ANA, antinuclear antibodies; BLyS, B lymphocyte stimulator; SLEDAI, Systemic Lupus Erythematosus Disease Activity Index; LN, lupus nephritis; NP SLE, neuropsychiatric systemic lupus erythematosus.

background standard of care therapy confounds the effects of investigational agents..$^{52,53}$

\section{Summary}

Caring for patients with systemic lupus erythematosus is a significant challenge. Rituximab seems to be a therapeutic option in cases of refractory diseases or recurrent flares despite the use of standard therapy. We cannot conclude that the promising results of open and multicenter-observational studies are more meaningful than results from double-blind placebo-controlled trials, but it would be unwise to reject the efficacy of an agent that targets a pathologic mechanism of the disease. We are in agreement that treatments should be more personalized and tailored case by case. For physicians who treat lupus patients, especially those patients with refractory disease who do not respond to standard treatment, data from open studies with rituximab can support the decision of the introduction of biologic therapy. As supported by offlabel use experience, rituximab seems to be more effective in refractory diseases than in nascent lupus nephritis. 
It may be necessary to conduct future research that defines the various biological mechanisms and genetic backgrounds that explain the varied manifestations of SLE and help predict response to treatment, allowing for more individualized or organ-specific therapies.

\section{Disclosure}

The authors report no conflict of interest in this work.

\section{References}

1. Rahman A, Isenberg DA. Systemic lupus erythematosus. N Engl J Med. 2008;358(9):929-939.

2. Tan EM, Cohen AS, Fries JF, et al. The 1982 revised criteria for the classification of systemic lupus erythematosus. Arthritis Rheum. 1982; 25(11):1271-1277.

3. Hochberg MC. Updating the American College of Rheumatology Criteria for the classification of systemic lupus erythematosus. Arthritis Rheum. 1997;40(9):1725.

4. Urowitz MB, Bookman AA, Koehler BE, Gordon DA, Smythe HA, Ogryzlo MA. The bimodal mortality pattern of systemic lupus erythematosus. Am J Med. 1976;60(2):221-225.

5. Wiesik-Szewczyk E, Lacki JK, Feleszko W, Olesinska M. Target therapies in systemic lupus erythematosus: current state of the art Mini Rev Med Chem. Sep 2010;10(10):956-965.

6. Merrill J. Ending the 50-year drought of FDA drug approval for SLE. Bull NYU Hosp Jt Dis. 2011;69(3):238-242.

7. Lipsky PE. Systemic lupus erythematosus: an autoimmune disease of B cell hyperactivity. Nat Immunol. 2001;2(9):764-766.

8. Anolik JH. B cell biology and dysfunction in SLE. Bull NYU Hosp Jt Dis. 2007;65(3):182-186.

9. Jacobi AM, Diamond B. Balancing diversity and tolerance: lessons from patients with systemic lupus erythematosus. J Exp Med. 2005; 202(3):341-344.

10. Yurasov S, Wardemann H, Hammersen J, et al. Defective B cell tolerance checkpoints in systemic lupus erythematosus. J Exp Med. 2005;201(5):703-711.

11. Arechiga AF, Habib T, He Y, et al. Cutting edge: the PTPN22 allelic variant associated with autoimmunity impairs B cell signaling. J Immunol. 2009;182(6):3343-3347.

12. Hom G, Graham RR, Modrek B, et al. Association of systemic lupus erythematosus with C8orf13-BLK and ITGAM-ITGAX. $N$ Engl J Med. 2008;358(9):900-909.

13. Liu Z, Davidson A. BAFF and selection of autoreactive B cells. Trends Immunol. 2011;32(8):388-394.

14. Nashi E, Wang Y, Diamond B. The role of B cells in lupus pathogenesis. Int J Biochem Cell Biol. 2010;42(4):543-550.

15. Silverman JG, Wiesman S. Rituximab therapy and autoimmune disorders: prospects for anti-B cell therapy. Arthritis Rheum. 2003; 48(6):1484-1492.

16. Anolik JH, Barnard J, Cappione A, et al. Ritiximab improves peripheral B cell abnormalities in human systemic lupus erythematosus. Arthritis Rheum. 2004;50(11):3580-3590.

17. Vallerskog T, Gunnarsson I, Widhe M, et al. Treatment with rituximab affects both the cellular and the humoral arm of the immune system in patients with SLE. Clin Immunol. 2007;122(1):62-74.

18. Anolik JH, Barnard J, Owen T, et al. Delayed memory B cell recovery in peripheral blood and lymphoid tissue in systemic lupus erythematosus after B cell depletion therapy. Arthritis Rheum. 2007;56(9): 3044-3056.

19. Vital EM, Dass S, Buch MH, et al. B cell biomarkers of rituximab responses in systemic lupus erythematosus. Arthritis Rheum. 2011; 63(10):3038-3047.
20. Merrill JT, Neuwelt CM, Wallace DJ, et al. Efficacy and safety of rituximab in moderately-to-severely active systemic lupus erythematosus: the randomized, double-blind, phase II/III systemic lupus erythematosus evaluation of rituximab trial. Arthritis Rheum. 2010;62(1):222-233.

21. Merrill J, Buyon J, Furie R, et al. Assessment of flares in lupus patients enrolled in a phase II/III study of rituximab (EXPLORER). Lupus. 2011;20(7):709-716.

22. Navarra SV, Guzman RM, Gallacher AE, et al; for BLISS-52 Study Group. Efficacy and safety of belimumab in patients with active systemic lupus erythematosus: a randomised, placebo-controlled, phase 3 trial. Lancet. 2011;377(9767):721-731.

23. Furie R, Petri M, Zamani O, et al; for BLISS-76 Study Group. A phase 3, randomized, placebo-controlled study of belimumab, a monoclonal antibody that inhibits B lymphocyte stimulator, in patients with systemic lupus erythematosus. Arthritis Rheum. 2011;63(12):3918-3930.

24. Furie RA, Petri MA, Wallace DJ, et al. Novel evidence-based systemic lupus erythematosus responder index. Arthritis Rheum. 2009;61(9): 1143-1151.

25. Rovin BH, Furie R, Latinis K, et al; for LUNAR Investigator Group. Efficacy and safety of rituximab in patients with active proliferative lupus nephritis: the Lupus Nephritis Assessment with Rituximab study. Arthritis Rheum. 2012;64(4):1215-1226.

26. Galarza-Maldonado C, Kourilovitch MR, Molineros JE, et al. The administration of low doses of rituximab followed by hydroxychloroquine, prednisone and low doses of mycophenolate mofetil is an effective therapy in Latin American patients with active systemic lupus erythematosus. Autoimmun Rev. 2010;10(2):108-111.

27. Catapano F, Chaudhry AN, Jones RB, Smith KG, Jayne DW. Longterm efficacy and safety of rituximab in refractory and relapsing systemic lupus erythematosus. Nephrol Dial Transplant. 2010;25(11): 3586-3592.

28. Leandro MJ, Cambridge G, Edwards JC, Ehrenstein MR, Isenberg DA. B-cell depletion in the treatment of patients with systemic lupus erythematosus: a longitudinal analysis of 24 patients. Rheumatology (Oxford). 2005;44(12):1542-1545.

29. Looney RJ, Anolik JH, Campbell D, et al. B cell depletion as a novel treatment for systemic lupus erythematosus: a phase I/II dose-escalation trial of rituximab. Arthritis Rheum. 2004;50(8):2580-2589.

30. Ng KP, Cambridge G, Leandro MJ, Edwards JC, Ehrenstein M, Isenberg DA. B cell depletion therapy in systemic lupus erythematosus: long-term follow-up and predictors of response. Ann Rheum Dis. 2007; 66(9):1259-1262.

31. Cambridge G, Isenberg DA, Edwards JC, et al. B cell depletion therapy in systemic lupus erythematosus: relationships among serum B lymphocyte stimulator levels, autoantibody profile and clinical response. Ann Rheum Dis. 2008;67(7):1011-1016.

32. Albert D, Dunham J, Khan S, et al. Variability in the biological response to anti-CD20 B cell depletion in systemic lupus erythematosus. Ann Rheum Dis. 2008;67(12):1724-1731.

33. Reynolds JA, Toescu V, Yee CS, Prabu A, Situnayake D, Gordon C. Effects of rituximab on resistant SLE disease including lung involvement. Lupus. 2009;18(1):67-73.

34. Tanaka Y, Yamamoto K, Takeuchi T, et al. A multicenter phase I/II trial of rituximab for refractory systemic lupus erythematosus. Mod Rheumatol. 2007;17(3):191-197.

35. Lu TY, Ng KP, Cambridge G, et al. A retrospective seven-year analysis of the use of B cell depletion therapy in systemic lupus erythematosus at University College London Hospital: the first fifty patients. Arthritis Rheum. 2009;61(4):482-487.

36. Garcia-Carrasco M, Mendoza-Pinto C, Sandoval-Cruz M, et al. Anti-CD20 therapy in patients with refractory systemic lupus erythematosus: a longitudinal analysis of 52 Hispanic patients. Lupus. 2010;19(2):213-219.

37. Terrier B, Amoura Z, Ravaud P, et al; for Club Rhumatismes et Inflammation. Safety and efficacy of rituximab in systemic lupus erythematosus: results from 136 patients from the French AutoImmunity and Rituximab registry. Arthritis Rheum. 2010;62(8):2458-2466. 
38. Jónsdóttir T, Gunnarsson I, Risselada A, Henriksson EW, Klareskog L, van Vollenhoven RF. Treatment of refractory SLE with rituximab plus cyclophosphamide: clinical effects, serological changes, and predictors of response. Ann Rheum Dis. 2008;67(3):330-334.

39. Gomard-Mennesson E, Ruivard M, Koenig M, et al. Treatment of isolated severe immune hemolytic anaemia associated with systemic lupus erythematosus: 26 cases. Lupus. 2006;15(4):223-231.

40. Lindholm C, Börjesson-Asp K, Zendjanchi K, Sundqvist AC, TarkowskiA, Bokarewa M. Longterm clinical and immunological effects of anti-CD20 treatment in patients with refractory systemic lupus erythematosus. J Rheumatol. 2008;35(5):826-833.

41. Díaz-Lagares C, Croca S, Sangle S, et al; for UK-BIOGEAS Registry. Efficacy of rituximab in 164 patients with biopsy-proven lupus nephritis: pooled data from European cohorts. Autoimmun Rev. 2012;11(5): $357-364$.

42. Lateef A, Lahiri M, Teng GG, Vasoo S. Use of rituximab in the treatment of refractory systemic lupus erythematosus: Singapore experience. Lupus. 2010;19(6):765-770.

43. Boletis JN, Marinaki S, Skalioti C, Lionaki SS, Iniotaki A, Sfikakis PP. Rituximab and mycophenolate mofetil for relapsing proliferative lupus nephritis: a long-term prospective study. Nephrol Dial Transplant. 2009;24(7):2157-2160.

44. Jónsdóttir T, Sundelin B, Welin Henriksson E, van Vollenhoven RF, Gunnarsson I. Rituximab-treated membranous lupus nephritis: clinical outcome and effects on electron dense deposits. Ann Rheum Dis. 2011; 70(6):1172-1173.

45. Melander C, Sallée M, Trolliet P, et al. Rituximab in severe lupus nephritis: early B-cell depletion affects long-term renal outcome. Clin J Am Soc Nephrol. 2009:4(3):579-587.
46. Jónsdóttir T, Gunnarsson I, Mourão AF, Lu TY, van Vollenhoven RF, Isenberg D. Clinical improvements in proliferative vs membranous lupus nephritis following B-cell depletion: pooled data from two cohorts. Rheumatology (Oxford). 2010;49(8):1502-1504.

47. Ramos-Casals M, Diaz-Lagares C, Soto-Cardenas MJ, et al. Rituximab therapy in lupus nephritis: current clinical evidence. Clin Rev Allergy Immunol. 2011;40(3):159-169.

48. Pepper R, Griffith M, Kirwan C, et al. Rituximab is an effective treatment for lupus nephritis and allows a reduction in maintenance steroids. Nephrol Dial Transplant. 2009;24(12):3717-3723.

49. Tokunaga M, Saito K, Kawabata D, et al. Efficacy of rituximab (antiCD20) for refractory systemic lupus erythematosus involving the central nervous system. Ann Rheum Dis. 2007;66(4):470-475.

50. Narváez J, Ríos-Rodriguez V, de la Fuente D, et al. Rituximab therapy in refractory neuropsychiatric lupus: current clinical evidence. Semin Arthritis Rheum. 2011;41(3):364-372.

51. Conti F, Perricone C, Ceccarelli F, Valesini G. Rituximab treatment of systemic lupus erythematosus in controlled trials and in clinical practice: Two sides of the same coin. Autoimmun Rev. 2010;9(11):716-720.

52. Bruce IN, Gordon C, Merrill JT, Isenberg D. Clinical trials in lupus: what have we learned so far? Rheumatology (Oxford). 2010;49(6): 1025-1027.

53. Merrill JT, Buyon JP. Rituximab: wanted dead or alive. Arthritis Rheum. 2010;62(8):2188-2191.
Biologics: Targets \& Therapy

\section{Publish your work in this journal}

Biologics: Targets \& Therapy is an international, peer-reviewed journal focusing on the patho-physiological rationale for and clinical application of Biologic agents in the management of autoimmune diseases, cancers or other pathologies where a molecular target can be identified. This journal is indexed on PubMed Central, CAS, EMBase, Scopus

\section{Dovepress}

and the Elsevier Bibliographic databases. The manuscript management system is completely online and includes a very quick and fair peerreview system, which is all easy to use. Visit http://www.dovepress com/testimonials.php to read real quotes from published authors. 